\title{
Hydrodynamic-based delivery of PTP1B shRNA reduces plasma glucose levels in diabetic mice
}

\author{
SANAZ VAKILI $^{1}$, SHADI SADAT SEYYED EBRAHIMI ${ }^{1}$, ASIE SADEGHI ${ }^{1}$, SATTAR GORGANI-FIRUZJAEE ${ }^{1}$, \\ MAANI BEIGY ${ }^{1}$, PARVIN PASALAR ${ }^{1}$ and REZA MESHKANI ${ }^{1,2}$ \\ ${ }^{1}$ Department of Biochemistry, Faculty of Medicine; ${ }^{2}$ Endocrinology and Metabolism Research Center, \\ Tehran University of Medical Sciences, Tehran, Iran
}

Received June 8, 2012; Accepted October 12, 2012

DOI: $10.3892 / \mathrm{mmr} .2012 .1172$

\begin{abstract}
Protein tyrosine phosphatase 1B (PTP1B) is a negative regulator of insulin signaling which is overexpressed in the liver of diabetic animals. The aims of this study were to generate liver-specific PTP1B knockout mice using a PTP1B-short hairpin RNA (shRNA) plasmid and to investigate the effect of PTP1B inhibition on plasma glucose levels in streptozotocin-induced diabetic mice. We first validated the hydrodynamic tail vein injection in mice using a vector carrying the luciferase gene. Expression of the PTP1B gene was quantified by real-time PCR. The level of phosphorylated Akt was examined by western blot analysis. The injection of the plasmid containing firefly luciferase revealed that the highest transfer of the vector into the liver was obtained $24 \mathrm{~h}$ after the injection of $20 \mu \mathrm{g}$ plasmid. The injection of PTP1B-shRNA, but not the scrambled shRNA plasmid, resulted in a reduction in PTP1B expression levels by up to $84 \%$ in the liver of the diabetic mice. Plasma glucose levels following the injection of PTP1B-shRNA remained significantly lower in the diabetic mice for 5 days. In addition, mice receiving PTP1B-shRNA in the basal and insulin-stimulated states had higher levels of Akt phosphorylation in the liver cells compared with mice that were injected with the scrambled sequence (35 and 60\%, respectively; $\mathrm{p}<0.01)$. Furthermore, PTP1B overexpression was observed in the muscle, liver, adipose, heart and kidney tissues of the diabetic mice. The data from this study demonstrate that PTP1B inhibition may be a promising approach for lowering plasma glucose levels in diabetic patients. However, further studies using non-viral carriers are required to deliver the plasmid safely into the liver.
\end{abstract}

Correspondence to: Dr Reza Meshkani, Department of Biochemistry, Faculty of Medicine, Tehran University of Medical Sciences, Keshavarz Blvd., Poursina St., 14176-13151 Tehran, Iran E-mail: rmeshkani@tums.ac.ir

Abbreviations: PTP1B, protein tyrosine phosphatase-1B; RNAi, RNA interference; shRNA, short hairpin RNA; STZ, streptozotocin

Key words: diabetes, protein tyrosine phosphatase 1B, hydrodynamic tail vein injection, liver-specific knockdown, RNA interference

\section{Introduction}

Protein tyrosine phosphatase 1B (PTP1B) is the main negative regulator of the insulin signaling pathway that has received significant attention over the last decade. This enzyme is widely expressed in insulin-sensitive tissues (1). PTP1B binds to the insulin receptor and efficiently dephosphorylates it in vitro. In vivo and in vitro studies have shown that changes in the expression of PTP1B are able to induce or prevent insulin resistance in muscle, adipose and liver tissues (2-6). Particularly, obese insulin-resistant and diabetic patients have demonstrated a higher expression and activity of PTP1B in their muscle and adipose tissues (7). The increased expression of PTP1B has also been reported in insulin-sensitive tissues from various animal models, including ob/ob mice, animals fed on high fat and fructose diets and streptozotocin (STZ)induced diabetic mice or rats $(6,8,9)$. Furthermore, in vitro studies have also shown that fatty acids, particularly palmitate, high glucose and cytokines, including tumor necrosis factor (TNF)- $\alpha$ and interleukin (IL)-6, are some of the factors that induce the expression of PTP1B in various tissues (10-13). Further studies using gene-targeting approaches have provided new insight into the role of PTP1B in the insulin signaling pathway. The tissue-specific overexpression of PTP1B in the liver and muscle has been shown to lead to a defect in insulin signaling, resulting in systemic insulin resistance in mice $(14,15)$. In addition, genetic variations of the PTP1B gene have been reported to be associated with hypertension, insulin resistance and type 2 diabetes in various populations (16-18).

Given the effect of PTP1B overexpression on the insulin signaling pathway, a number of attempts have been directed toward reducing PTP1B expression levels in liver, muscle and adipose tissues. PTP1B knockout mice indicated an increased phosphorylation of the insulin receptor in the liver and muscle tissues following insulin injection $(3,4)$. Notably, PTP1B-deficient mice are resistant to weight gain, exhibit increased insulin sensitivity and remain insulin-sensitive when subjected to a high-fat diet (3). Also, liver-specific PTP1B knockout mice have demonstrated improved insulin sensitivity and decreased plasma glucose and insulin levels (19). In addition, PTP1B-deficient myocytes have demonstrated increased insulin sensitivity and protection against TNF- $\alpha$-induced insulin resistance (5). The results from these studies suggest that 
decreasing the expression and activity of PTP1B is potentially a therapeutic approach for treating individuals with metabolic syndrome and type 2 diabetes.

A variety of strategies, including antisense oligonucleotides, chemical inhibitors and RNA interference (RNAi), have been used to inhibit PTP1B expression and activity in in vitro and in vivo studies. The decreased expression of PTP1B achieved using antisense oligonucleotides has been shown to lead to increased insulin receptor tyrosine phosphorylation and insulin sensitivity in monkeys (20). A number of research groups have also attempted to identify specific inhibitors capable of decreasing PTP1B activity in vitro $(21,22)$. However, given the similarities among the phosphatases, these studies have not been successful in providing a specific PTP1B inhibitor for in vivo investigation. RNAi has been introduced as a promising approach for the treatment of certain metabolic diseases. A certain study revealed that the hydrodynamic injection of PTP1B-small interfering RNA (siRNA) led to a decrease in the plasma glucose levels of diabetic mice (23). However, due to the short-term effects of synthetic siRNA, serious challenges have been encountered in in vivo studies. Short hairpin RNA (shRNA) is a member of the RNAi family and, when its sequence is inserted into bacterial or viral plasmids, it enables the shRNA to be expressed more potently. In this study, we used PTP1B-shRNA to knockdown PTP1B expression in the liver of diabetic mice using a hydrodynamic tail vein injection technique. Although this procedure is not applicable to humans, it is useful in primary studies where the liver is the target tissue. To our knowledge, no previous studies have explored the behavior of shRNA specific for the knockdown of PTP1B in the liver of diabetic mice. Therefore, the aims of this study were to generate liver-specific PTP1B knockout mice using PTP1B-shRNA and to investigate the effects of the PTP1B knockdown on plasma glucose and lipid levels in STZ-induced diabetic mice.

\section{Materials and methods}

Plasmids. The pGL3-promoter plasmid containing firefly luciferase and the pRS plasmid containing PTP1B-shRNA were purchased from OriGene Technologies, Inc. (Rockville, MD, USA). The PTP1B shRNA sequence (AATTGCACC AGGAAGATAATGACTATATC) was selected based on our previous study (24). A scrambled sequence shRNA-vector was also used as the control. Following transfection of the 2 plasmids into $E$. coli, they were cultured in LB overnight and then the plasmids were extracted using the Maxi kit of Qiagen (Valencia, CA, USA). The purity of the plasmid preparations was determined by $1 \%$ agarose gel electrophoresis. The assay kit for determining luciferase activity was purchased from Promega (Madison, WI, USA).

Animal care and treatment. A total of 18 male NMRI mice weighing 15-18 g (Pasteur Institute, Tehran, Iran) were housed in individual cages and maintained under controlled conditions of temperature $\left(24 \pm 3^{\circ} \mathrm{C}\right)$ and light (12-h light/dark cycle) and fed a standard rodent chow and water ad libitum. The mice were divided into 2 groups and received 10 or $20 \mu \mathrm{g}$ plasmid DNA (pGL3-Luc) via the hydrodynamic tail vein injection method. Each group was then separately divided into 3 groups for the measurement of luciferase activity at different inter- vals $(8,16$ and $24 \mathrm{~h})$. The animals were sacrificed following ketamine-xylazine anesthesia. After collecting the whole-body blood, liver, lung, heart, kidney, muscle, intestine and adipose tissue were dissected and frozen in liquid nitrogen. The tissues were stored at $-80^{\circ} \mathrm{C}$ for further analysis.

Luciferase studies. The luciferase activity of the pGL3-promoter plasmid was assessed in protein tissue extracts using a luminometer. Cold tissue lysis buffer $(1 \mathrm{ml}$; $0.1 \mathrm{M}$ Tris-HCl, 2 mM EDTA, $0.1 \%$ Triton $\mathrm{X}-100,0.1 \mathrm{mM}$ phenylmethylsulfonyl fluoride and $1 \mathrm{X}$ protease inhibitor cocktail, $\mathrm{pH} 7.8$ ) was added to $30 \mathrm{mg}$ of each tissue, which was then homogenized and centrifuged in a microcentrifuge for $10 \mathrm{~min}$ at $13000 \mathrm{x} \mathrm{g}$ at $4^{\circ} \mathrm{C}$. The protein concentration of the supernatant was determined using the Bradford method. The supernatant of the homogenate $(10 \mu \mathrm{l})$ was mixed with $100 \mathrm{ml}$ luciferase assay reagent and the luciferase activity was measured using a luminometer. The firefly luciferase activity was normalized to the total amount of protein.

Hydrodynamic injection of PTPIB-shRNA into diabetic mice. A total of 30 male NMRI mice weighing 15-18 g were divided into 2 groups and allocated to separate cages. The first group, the non-diabetic control $(n=10)$, received Na-citrate buffer and the second group $(\mathrm{n}=20)$ was injected with $50 \mathrm{mg} / \mathrm{kg} \mathrm{STZ}$ (Sigma, St. Louis, MO, USA) for 5 consecutive days based on a low-dose STZ induction protocol. Body weight and glucose levels were assessed during the study for 5 weeks prior to the hydrodynamic injection. Plasma glucose concentrations were measured using a glucometer (Accu-Check Active; Roche Diagnostic Corporation, Mannheim, Germany) after fasting for $6 \mathrm{~h}$. After 4-5 weeks, those mice with blood glucose levels $>200 \mathrm{mg} / \mathrm{dl}$ were selected and divided into 2 groups, the diabetic control and diabetic groups. The diabetic control mice were injected with the scrambled shRNA sequence, while the diabetic group mice were injected with $20 \mu \mathrm{g}$ plasmid pRS (PTP1B-shRNA) via hydrodynamic tail vein injection. The plasma glucose concentration was monitored during the week following the injection. To investigate the insulin signaling pathway in the liver of the animals, 1 unit of insulin was injected intravenously into some animals of all groups. Various tissues of the diabetic mice, including liver, lung, heart, kidney, muscle and adipose tissue, were dissected after collecting the whole-body blood. Non-diabetic control mice $(n=10)$ also received an injection of the PTP1B-shRNA and the scrambled shRNA sequence. All procedures and protocols were performed in accordance with the institutional guidelines for animal care which were approved by the ethics committee of Tehran University of Medical Sciences.

Gene expression analysis. Total RNA was extracted from certain tissues, including liver, lung, heart, kidney, muscle and adipose tissues, from all groups (non-diabetic, diabetic, diabetic receiving the scrambled shRNA and diabetic receiving PTP1B-shRNA) using QIAzol. Total RNA (1 $\mu \mathrm{g})$ was reverse transcribed using Qiagen reverse transcriptase and random hexamer primer. Real-time PCR was performed on a RotorGene 3000 instrument (Corbett Research, Sydney, Australia). PTP1B expression levels were assessed by a QuantiTect primer assay for PTP1B using QuantiFast SYBR-Green PCR master mix. The data were normalized against the $\beta$-actin transcript level. The amplification protocol for 40 cycles was as follows: $5 \mathrm{~min}$ 


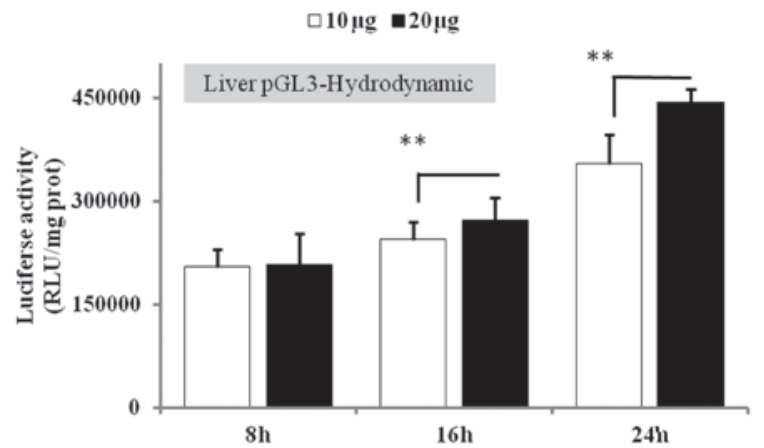

Figure 1. Luciferase activity in the liver 8,16 and $24 \mathrm{~h}$ post injection of 10 or $20 \mu \mathrm{g}$ pGL3-promoter plasmid using hydrodynamic gene transfer. ${ }^{* *} \mathrm{p}<0.001$ for the comparison between 10 and $20 \mu \mathrm{g}$ of the pGL3-promoter plasmid.

at $95^{\circ} \mathrm{C}$ for initial activation, $10 \mathrm{sec}$ at $95^{\circ} \mathrm{C}$ for denaturation and $30 \mathrm{sec}$ at $60^{\circ} \mathrm{C}$ for annealing/extension.

Western blot analysis. A tissue lysate was prepared by homogenization in modified RIPA buffer (50 mM Tris- $\mathrm{HCl} \mathrm{pH} 7.4$, $1 \%$ Triton $\mathrm{X}-100,0.2 \%$ sodium deoxycholate, $0.2 \%$ SDS, $1 \mathrm{mM}$ Na-EDTA and $1 \mathrm{mM}$ PMSF) supplemented with protease inhibitor cocktail (Roche, Mannheim, Germany). For detection of phosphoprotein, a buffer consisting of $50 \mathrm{mM}$ HEPES $\mathrm{pH}$ 7.5, $150 \mathrm{mM} \mathrm{NaCl}, 100 \mathrm{mM} \mathrm{NaF}, 10 \mathrm{mM}$ EDTA, $10 \mathrm{mM}$ $\mathrm{Na}_{4} \mathrm{P}_{2} \mathrm{O}_{7}, 2 \mathrm{mM} \mathrm{NaVO}$ and protease inhibitor cocktail was used. The protein concentration was determined using the Bradford method. The total protein $(20-30 \mathrm{mg}$ ) was fractionated by SDS-PAGE and the gel was transferred onto a PVDF membrane (Millipore, Schwalbach, Germany). The membrane was blocked overnight in blocking buffer $(5 \%$ skimmed milk in TBST buffer) and then incubated for $1 \mathrm{~h}$ with primary antibodies diluted in TBST containing 1\% BSA. The primary antibodies used were as follows: Akt and phospho-Akt (Ser473) (Cell Signaling Technology, Beverly, MA, USA) and $\beta$-actin (Abcam, Cambridge, MA, USA). The membrane was then incubated with secondary antibody conjugated to HRP (Santa Cruz Biotechnology, Santa Cruz, USA) for $1 \mathrm{~h}$ and detection was performed using ECL reagents (Amersham Pharmacia Corp., Piscataway, NJ, USA). The films were scanned and protein bands were quantified using Scion Image software. Each experiment was performed at least 3 times.

Measurements of clinical biochemistry parameters. The concentrations of aspartate transaminase (AST), alanine transaminase (ALT), lactate dehydrogenase (LDH), uric acid, albumin and total protein were measured using commercial kits to analyze the serum of diabetic mice receiving either the scrambled or PTP1B-shRNA, and in that of the normal mice that did not receive any injection, $24 \mathrm{~h}$ post-injection. The levels of triglyceride, LDL, cholesterol and HDL were measured 1 week after hydrodynamic injection.

Statistical analysis. Data are expressed as the means \pm SE and were analyzed using SPSS 13.0 (SPSS, Inc., Chicago, IL, USA). One-way ANOVA was used for statistical analysis to determine differences between groups. The independent t-test was performed when a statistical significance was found. A p-value $<0.05$ was considered to indicate a statistically significant result.

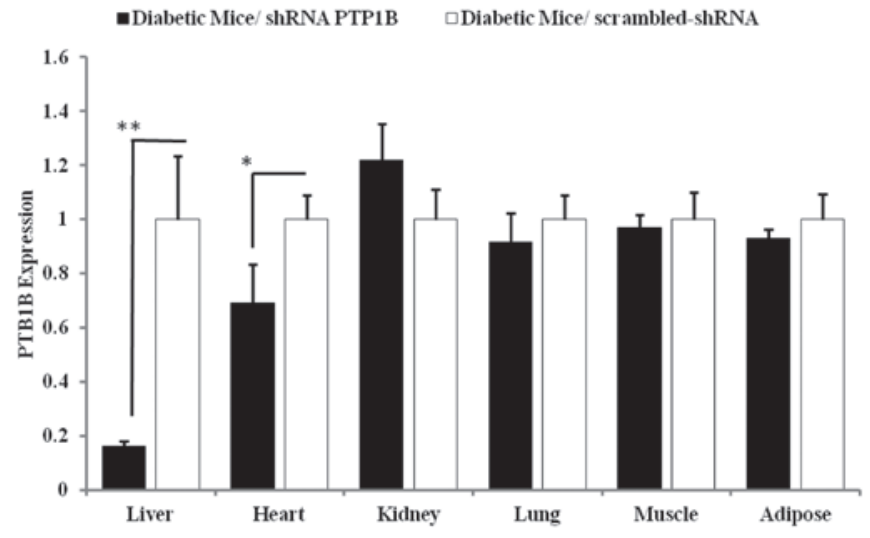

Figure 2. PTP1B expression in various tissues of streptozotocin-induced diabetic mice following the hydrodynamic tail vein injection of $20 \mu \mathrm{g}$ pRSPTP1B-shRNA compared with the scrambled-shRNA group $(n=6)$. Real-time PCR was performed on liver, heart, kidney, lung, muscle and adipose extracts of the mice. A marked and significant difference is specifically observed in the hepatic PTP1B expression levels of the diabetic mice receiving PTP1B-shRNA compared with those receiving scrambled shRNA $\left({ }^{*} \mathrm{p}<0.01,{ }^{* * *} \mathrm{p}<0.001\right)$.

\section{Results}

Optimization of the dose and time of in vivo gene expression following hydrodynamic injection. To validate the hydrodynamic-based procedure as an appropriate method for the specific delivery of the gene of interest into the liver, we first set up experiments to determine a suitable dose and time of luciferase gene expression in the liver. Luciferase gene activity was measured in various tissues 8,16 and 24 h post-injection of the plasmid at doses of 10 and $20 \mu \mathrm{g}$. The results revealed no significant difference between the 2 doses $8 \mathrm{~h}$ after injection, but, at 16 and $24 \mathrm{~h}$ post-injection, an efficient and specific luciferase activity was observed in the liver (data not shown). However, $20 \mu \mathrm{g}$ plasmid after $24 \mathrm{~h}$ had greater luciferase activity $(19.5 \%, \mathrm{p}<0.001)$ than $10 \mu \mathrm{g}$ at $24 \mathrm{~h}$ (Fig. 1). According to these data, we selected $20 \mu \mathrm{g}$ plasmid at $24 \mathrm{~h}$ to study the effect of PTP1B knockdown on gene expression analysis.

Liver-specific knockdown of PTP1B by shRNA in diabetic mice. To confirm that the hydrodynamic tail vein injection of $20 \mu \mathrm{g}$ PTP1B-shRNA led to a specific decrease of PTP1B expression in the liver, the mRNA levels of PTP1B were quantified in various tissues. As shown in Fig. 2, PTP1B-shRNA injection significantly reduced PTP1B expression in the liver of the mice by up to $84 \%(\mathrm{p}<0.0001)$ compared with the control group receiving the scrambled shRNA. No significant differences were identified in the levels of PTP1B expressed in the muscle, adipose, lung and kidney tissues of the mice compared with the controls, whereas the heart exhibited a $30 \%$ reduction of PTP1B expression following PTP1B-shRNA injection. These findings demonstrate that PTP1B-shRNA is able to decrease PTP1B expression somewhat specifically in the liver.

Effects of liver-specific knockdown on glucose and lipid parameters. We then aimed to evaluate whether hepatic PTP1B inhibition could be an efficient and specific strategy to reduce hyperglycemia in diabetic mice. All diabetic mice with plasma glucose levels $>200 \mathrm{mg} / \mathrm{dl}$ received either $20 \mu \mathrm{g}$ pRS 


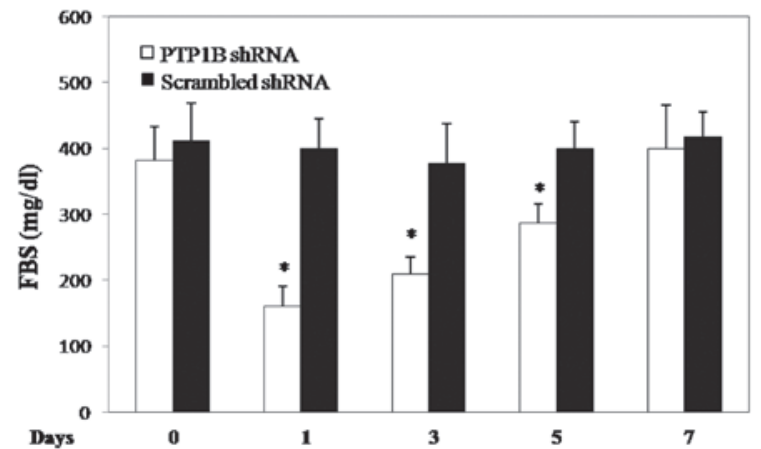

Figure 3. Plasma glucose levels in diabetic mice. Plasma glucose levels were measured at different time-points following the injection of $20 \mu \mathrm{g}$ PTP1BshRNA plasmid or scrambled-shRNA in diabetic mice. ${ }^{*} \mathrm{p}<0.001$ vs. day 0 in mice receiving PTP1B-shRNA.
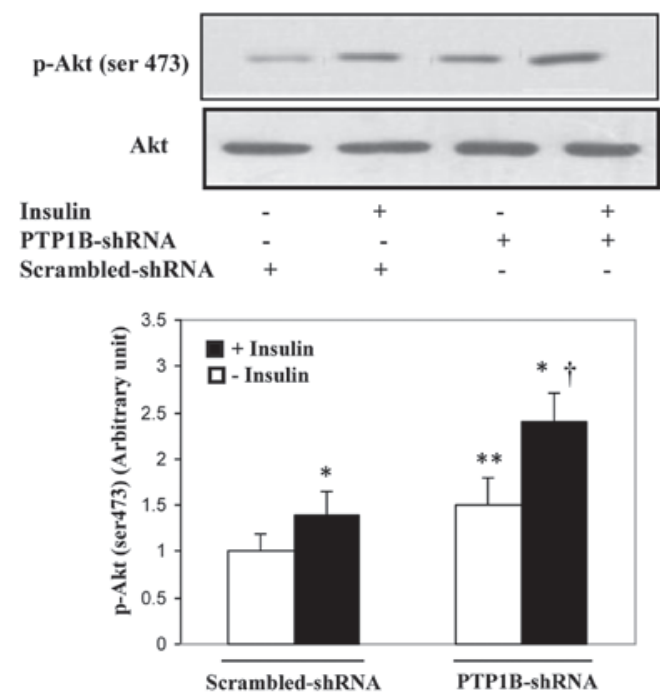

Figure 4. Effect of PTP1B inhibition in the liver on Akt phosphorylation. Before dissecting, mice were injected with 1 unit insulin for $15 \mathrm{~min}$. Liver extract lysates were subjected to western blot analysis using specific antibodies. The figure shows representative data gained from 3 independent animals (mean $\pm \mathrm{SE}$ ). ${ }^{*}<<0.001$ vs. in the absence of insulin; ${ }^{* *} \mathrm{p}<0.001$ vs. scrambled shRNAtreated mice in the absence of insulin; ${ }^{\dagger} \mathrm{p}<0.001 \mathrm{vs}$. scrambled shRNA-treated mice in the presence of insulin.

(PTP1B-shRNA) plasmid or the scrambled shRNA vector. At 1 day post-injection, the plasma glucose concentration markedly decreased by up to $58 \%(382 \pm 51$ vs. $160.8 \pm 31 \mathrm{mg} / \mathrm{dl}, \mathrm{n}=6$, p $<0.0001$; Fig. 3) in diabetic mice, while no significant changes were observed in the mice receiving the scrambled shRNA vector. On days $3(45 \%)$ and $5(25 \%)$ the plasma glucose levels remained significantly lower than on the day of injection of PTP1B-shRNA; however, there was no significant difference in the plasma glucose levels 1 week after PTP1B-shRNA injection. These findings demonstrate a significant effect of PTP1B inhibition on the plasma glucose levels for a few days in diabetic mice. No significant difference in the lipid profile (triglyceride, cholesterol, HDL and LDL) was observed between the diabetic and non-diabetic mice (data not shown). An injection of PTP1B-shRNA or the scrambled shRNA did not change the lipid levels of the diabetic mice.

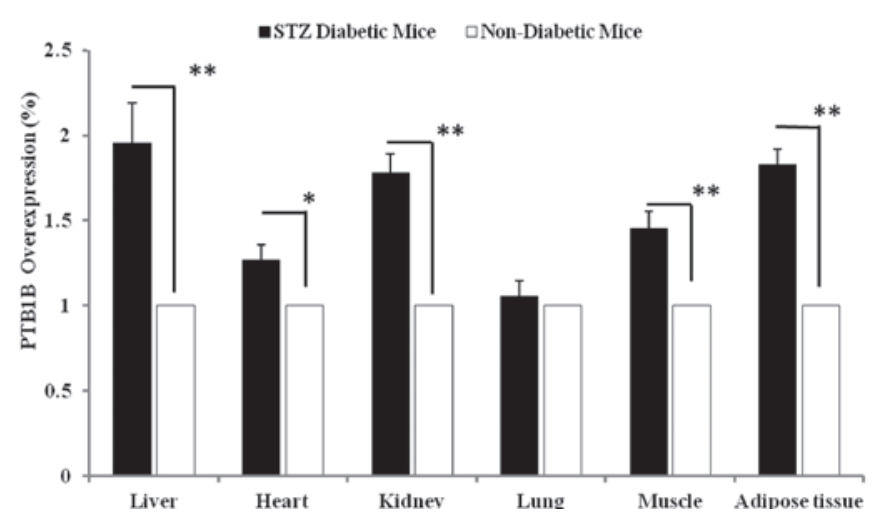

Figure 5. PTP1B expression in various tissues of healthy and streptozotocininduced diabetic mice. No hydrodynamic injection was performed in these groups of mice $(n=6)$. Real-time PCR data were obtained from liver, heart, kidney, lung, muscle and adipose extracts of the mice. ${ }^{*} \mathrm{p}<0.01,{ }^{* *} \mathrm{p}<0.001$ between the diabetic and non-diabetic mice.

Enhancement of hepatic insulin signaling in mice receiving PTPIB-shRNA. To investigate whether the decreased plasma glucose levels in diabetic mice receiving PTP1B-shRNA were at least partly due to the enhancement of insulin signaling activity, the phosphorylation of the key element of this pathway (Akt, Ser473) was studied in the liver. As shown in Fig. 4, the results demonstrated that insulin induced the phosphorylation of Akt in the PTP1B-shRNA- and scrambled-shRNA-treated mice by 70 and $50 \%$, respectively $(\mathrm{p}<0.001)$. In addition, mice receiving PTP1B-shRNA in the basal and insulin-stimulated states had higher Akt phosphorylation levels in the liver cells than the corresponding mice that were injected with the scrambled sequence (35 and 60\%, respectively; $\mathrm{p}<0.01$ ).These findings suggest that PTP1B inhibition in the liver results in insulin sensitivity leading to decreased plasma glucose levels in diabetic mice.

Effect of diabetes on PTP1B expression in various tissues. We assessed the PTP1B expression levels in various tissues of the healthy and diabetic mice. No injection was administered to these animals. As shown in Fig. 5, 3 insulin-sensitive tissues (liver, muscle and adipose) significantly demonstrated the elevated levels of PTP1B expression in the diabetic mice compared with those of the healthy group. The PTP1B gene demonstrated a 95\% (p<0.001), 45\% (p<0.001) and $82.0 \%$ $(\mathrm{p}<0.001)$ overexpression in liver, muscle and adipose, respectively. These data also demonstrate the overexpression of PTP1B in the kidney $(78 \%, \mathrm{p}<0.001)$ and heart $(26.6 \%, \mathrm{p}<0.01)$ in the diabetic mice compared with the controls.

Metabolic profile in diabetic and healthy mice. To investigate the effect of the hydrodynamic tail vein injection on liver function, several parameters were assessed. The concentrations of hepatic enzymes, albumin, uric acid and total protein were measured in the serum of mice receiving either the scrambled or PTP1B-shRNA and in that of the normal mice that did not receive any injection (data not shown). The levels of ALT and AST were 10 -fold $(\mathrm{p}<0.001)$ higher than those in normal mice following hydrodynamic injection. In the case of $\mathrm{LDH}$, a marked increase of 172 -fold $(\mathrm{p}<0.001)$ was observed at $24 \mathrm{~h}$ post-injection compared with the normal mice. The concentrations of albumin and total protein were reduced by 


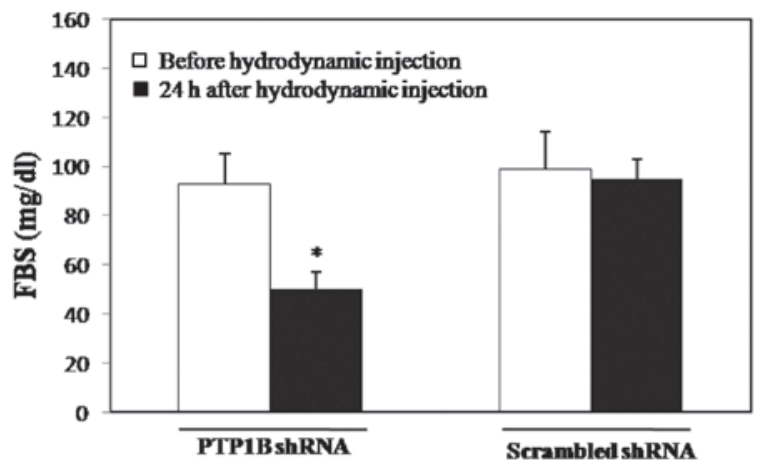

Figure 6. Plasma glucose levels prior to and following hydrodynamic injection in non-diabetic mice. $20 \mu \mathrm{g}$ PTP1B-shRNA or scrambled shRNA plasmids were injected and the plasma glucose levels were measured after $24 \mathrm{~h}(\mathrm{n}=6) .{ }^{*} \mathrm{p}<0.001 \mathrm{vs}$. before hydrodynamic injection.

1.2- and 1.5-fold ( $\mathrm{p}<0.001)$, respectively, compared with those in normal animals. These altered parameters tended to return to normal levels after 1 week (25).

Liver-specific knockdown of PTPIB by shRNA in healthy mice. We revealed that the PTP1B gene was inhibited by PTP1B-shRNA, even in healthy animals, $24 \mathrm{~h}$ post-hydrodynamic tail vein injection. In particular, the plasma glucose level was decreased by $46 \%$ in the $6-\mathrm{h}$ fasted mice $(93.5 \pm 8$ vs. $50.5 \pm 9 \mathrm{mg} / \mathrm{dl}, \mathrm{n}=6, \mathrm{p}<0.001$; Fig. 6). These animals remained healthy and no hypoglycemic symptoms were observed until $24 \mathrm{~h}$ post-injection.

\section{Discussion}

There is increasing evidence supporting the fact that type 2 diabetes is now a serious health problem all over the world. During the last decade, a number of cellular and molecular aspects of the diseases have been recognized, and these have aided researchers in the search for therapeutic targets for the treatment of diabetes. Evidence from human and animal studies support PTP1B as a new candidate in the field of diabetes. The increased expression of PTP1B in various tissues of diabetic patients is a major concern, and strategies based on the inhibition of PTP1B may have a beneficial effect on diabetes-associated complications. Numerous efforts have been made to identify specific chemical inhibitors of PTP1B for use in diabetes treatment. However, they face many difficulties as PTP1B resembles other phosphatases, including leukocyte antigen-related (LAR), SH2-containing inositol 5-phosphatase 2 (SHIP2) and T-cell protein tyrosine phosphatase or PTN2 (TC-PTP). The use of oligonucleotides is another strategy under investigation and a few studies have used antisense oligonucleotides and siRNA against PTP1B to investigate different aspects of the disease in animal models $(20,23)$. However, none of the studies have used shRNA against PTP1B and the present study is the first to apply PTP1B-shRNA in the treatment of diabetes. shRNA is superior to siRNA since it may be expressed more stably.

The liver is a vital organ in vertebrates that plays a central role in coordinating the whole body metabolism, including carbohydrate, lipid and protein metabolism, xenobiotic metabolism and detoxification (26). PTP1B overexpression in liver has been reported to be significant in the development of whole body insulin resistance in various animal models (14). Therefore, inhibition of PTP1B expression appears to have a beneficial effect on insulin resistance and diabetes. In this study we generated liver-specific PTP1B knockdown mice using shRNA by a hydrodynamic tail vein injection procedure. This method has been previously shown to deliver transgenes (pCMV-Luc, pGL3 and pSHAG-664) specifically into the liver $(23,27)$. We first attempted to validate this procedure in our study by luciferase gene delivery. Our results confirm that the hydrodynamic tail vein injection is a suitable method for delivering the gene of interest specifically into the liver. According to the results of our validation assay, we selected $20 \mu \mathrm{g}$ plasmid (pRS-PTP1B-shRNA) for the following steps of the study. The injection of $20 \mu \mathrm{g}$ plasmid (pRS-PTP1BshRNA) via the tail vein led to a marked decrease in plasma glucose levels in the diabetic and non-diabetic mice, reduced the expression of PTP1B specifically in the liver and increased the sensitivity of the insulin signaling pathway in the liver.

The glucose lowering effect of PTP1B inhibition in the liver is the main finding of this study. Notably, a single injection of $20 \mu \mathrm{g}$ PTP1B-shRNA plasmid resulted in a 58\% decrease in plasma glucose levels after $24 \mathrm{~h}$. Although the plasma glucose levels tended to gradually increase on days 3 and 5, the animals that received PTP1B-shRNA had significantly lower glucose levels than those that received scrambled-shRNA on those days. The same results were also observed in non-diabetic mice. These results are in the line with previous reports that PTP1B inhibition leads to a reduction in plasma glucose levels in animal models $(3,5,20,23)$. For example, PTP1B knockout mice are not only insulin-sensitive but also maintain euglycemia in the fed state (3). In another study, the hydrodynamic injection of PTP1B-siRNA led to a decrease in the plasma glucose levels of diabetic mice only for $24 \mathrm{~h}$, whereas, in this study, PTP1BshRNA reduced glucose levels for 5 days (23). The short-term effect of PTP1B-siRNA on the glucose levels of diabetic mice may be attributed to the nature of the siRNA. Although siRNA delivery to the cytosol should be easier to achieve compared with the delivery of shRNA, which must enter the nucleus and undergo transcription, the shRNA has been established as an improved substrate for dicer and exhibits improved RISC loading. It has been previously demonstrated that plasmid-based shRNA is much more potent than siRNA (28). It has also been suggested that a significant amount of siRNA is lost to premature metabolism following hydrodynamic administration (29). It is noteworthy that PTP1B antisense oligonucleotides have been investigated for ability to normalize blood glucose levels in phase II clinical trials (30).

To confirm that the decreased plasma glucose levels in diabetic mice are due to the inhibition of PTP1B expression in the liver, expression analysis was performed. The data demonstrated that among the insulin-sensitive tissues (liver, muscle and adipose), the liver was the only one that had a reduction in the expression of PTP1B. An investigation of the phosphorylation of Akt also provided further evidence on the role of PTP1B in the insulin signaling pathway. The results demonstrated that in the liver, PTP1B has a major effect on glucose homeostasis and its reduced expression led to insulin sensitivity in liver resulting in decreased plasma glucose levels in the mice. However, in the present study, we did not observe a difference in the lipid 
profile (triglyceride, cholesterol, LDL and HDL cholesterol) between the diabetic and healthy mice. The lack of a change in the lipid profile may be due to the method of diabetes induction (low-dose STZ) in this study. Corresponding results were also reported in another study in which low-dose STZ injection was used to induce diabetes in mice (31).

In this study, we also investigated the expression of PTP1B in various tissues of the diabetic mice. In addition to increased expression levels of PTP1B in the liver, muscle and adipose tissues, the observation of enhanced expression of PTP1B in the heart and kidney is unique and it is the first time that such results have been reported. In addition, with respect to the hydrodynamic procedure (1) we assessed the levels of hepatic enzymes and certain other proteins in the diabetic animals. According to the data, the liver showed symptoms of primary damage due to the high pressure of the hydrodynamic injection which is understandable from the high levels of ALT, AST and LDH. However, these abnormal factors appeared to reverse with time and the levels of the parameters returned to normal values after a few days.

In conclusion, the data from this study provide evidence that PTP1B inhibition by PTP1B-shRNA administration may be a promising approach for lowering plasma glucose levels in diabetic patients. The study used hydrodynamic tail vein injection but the application of this method to humans is not possible. Therefore, the targeting of the PTP1B-shRNA to the liver using non-viral carriers may be a subject for investigation in further studies.

\section{References}

1. Goldstein BJ: Regulation of insulin receptor signaling by protein-tyrosine dephosphorylation. Receptor 3: 1-15, 1993.

2. Zabolotny JM, Haj FG, Kim YB, Kim HJ, et al: Transgenic overexpression of protein-tyrosine phosphatase 1B in muscle causes insulin resistance, but overexpression with leukocyte antigen-related phosphatase does not additively impair insulin action. J Biol Chem 279: 24844-24851, 2004.

3. Elchebly M, Payette P, Michaliszyn E, et al: Increased insulin sensitivity and obesity resistance in mice lacking the protein tyrosine phosphatase-1B gene. Science 283: 1544-1548, 1999.

4. Klaman LD, Boss O, Peroni OD, et al: Increased energy expenditure, decreased adiposity, and tissue-specific insulin sensitivity in protein-tyrosine phosphatase 1B-deficient mice. Mol Cel Biol 20: 5479-5489, 2000.

5. Nieto-Vazquez I, Fernández-Veledo S, de Alvaro C, Rondinone CM, Valverde AM and Lorenzo M: Protein-tyrosine phosphatase 1B-deficient myocytes show increased insulin sensitivity and protection against tumor necrosis factor-alpha-induced insulin resistance. Diabetes 56: 404-413, 2007.

6. Dadke SS, Li HC, Kusari AB, Begum N and Kusari J: Elevated expression and activity of protein-tyrosine phosphatase $1 \mathrm{~B}$ in skeletal muscle of insulin-resistant type II diabetic Goto-Kakizaki rats. Biochem Biophys Res Commun 274: 583-589, 2000.

7. Ahmad F, Azevedo JL, Cortright R, Dohm GL and Goldstein BJ: Alterations in skeletal muscle protein-tyrosine phosphatase activity and expression in insulin-resistant human obesity and diabetes. J Clin Invest 100: 449-458, 1997.

8. Wu Y, Ouyang JP, Wu K, Wang SS, Wen CY and Xia ZY: Rosiglitazone ameliorates abnormal expression and activity of protein tyrosine phosphatase $1 \mathrm{~B}$ in the skeletal muscle of fat-fed, streptozotocin-treated diabetic rats. Br J Pharmacol 146 234-243, 2005

9. Adeli K, Macri J, Mohammadi A, Kito M, Urade R and Cavallo D: Apolipoprotein B is intracellularly associated with an ER-60 protease homologue in HepG2 cells. J Biol Chem 272: 22489-22494, 1997.
10. Zabolotny JM, Kim YB, Welsh LA, Kershaw EE, Neel BG and Kahn BB: Protein-tyrosine phosphatase 1B expression is induced by inflammation in vivo. J Biol Chem 283: 14230-14241, 2008.

11. Parvaneh L, Meshkani R, Bakhtiyari S, et al: Palmitate and inflammatory state additively induce the expression of PTP1B in muscle cells. Biochem Biophys Res Commun 396: 467-71, 2010.

12. MohammadTaghvaei N, Meshkani R, Taghikhani M, Larijani B and Adeli K: Palmitate enhances protein tyrosine phosphatase 1B (PTP1B) gene expression at transcriptional level in $\mathrm{C} 2 \mathrm{C} 12$ skeletal muscle cells. Inflammation 34: 43-48, 2011.

13. Wu J, Yang LJ, Zou DJ: Rosiglitazone attenuates tumor necrosis factor- $\alpha$-induced protein-tyrosine phosphatase-1B production in HepG2 cells. J Endocrinol Invest 35: 28-34, 2012.

14. Haj FG, Zabolotny JM, Kim YB, Kahn BB and Neel BG: Liver-specific protein-tyrosine phosphatase 1B (PTP1B) re-expression alters glucose homeostasis of $\mathrm{PTP} 1 \mathrm{~B}^{-/-}$mice. $\mathrm{J}$ Biol Chem 280: 15038-15046, 2005.

15. Delibegovic M, Bence KK, Mody N, et al: Improved glucose homeostasis in mice with muscle-specific deletion of protein-tyrosine phosphatase 1B. Mol Cell Biol 27: 7727-7734, 2007.

16. Gu P, Jiang W, Du H, Shao J, Lu B, Wang J and Zou D: Protein tyrosine phosphatase $1 \mathrm{~B}$ gene polymorphisms and essential hypertension: a case-control study in Chinese population. J Endocrinol Invest 33: 483-488, 2010.

17. Meshkani R, Taghikhani M, Al-Kateb H, Larijani B, et al: Polymorphisms within the protein tyrosine phosphatase $1 \mathrm{~B}$ (PTPN1) gene promoter: functional characterization and association with type 2 diabetes and related metabolic traits. Clin Chem 53: 1585-1592, 2007.

18. Meshkani R, Taghikhani M, Mosapour A, et al: 1484insG polymorphism of the PTPN1 gene is associated with insulin resistance in an Iranian population. Arch Med Res 38: 556-562, 2007.

19. Delibegovic M, Zimmer D, Kauffman C, et al: Liver-specific deletion of protein-tyrosine phosphatase 1B (PTP1B) improves metabolic syndrome and attenuates diet-induced endoplasmic reticulum stress. Diabetes 58: 590-599, 2009.

20. Swarbrick MM, Havel PJ, Levin AA, et al: Inhibition of protein tyrosine phosphatase-1B with antisense oligonucleotides improves insulin sensitivity and increases adiponectin concentrations in monkeys. Endocrinology 150: 1670-1679, 2009.

21. Koren S and Fantus IG: Inhibition of the protein tyrosine phosphatase PTP1B: potential therapy for obesity, insulin resistance and type-2 diabetes mellitus. Best Pract Res Clin Endocrinol Metab 21: 621-40, 2007

22. Zhang S and Zhang ZY: PTP1B as a drug target: recent developments in PTP1B inhibitor discovery. Drug Discov Today 12: 373-381, 2007.

23. Xu J, Li L, Hong J and Huang W: Effects of small interference RNA against PTP1B and TCPTP on insulin signaling pathway in mouse liver: evidence for non-synergetic cooperation. Cell Biol Int 31: 88-91, 2007.

24. Bakhtiyari S, Meshkani R, Taghikhani M, Larijani B and Adeli K: Protein tyrosine phosphatase-1B (PTP-1B) knockdown improves palmitate-induced insulin resistance in $\mathrm{C} 2 \mathrm{C} 12$ skeletal muscle cells. Lipids 45: 237-244, 2010.

25. Maruyama H, Higuchi N, Nishikawa Y, et al: High level expression of naked DNA delivered to rat liver via tail vein injection. J Gene Med 4: 333-341, 2002.

26. Meshkani R and Adeli K: Hepatic insulin resistance, metabolic syndrome and cardiovascular disease. Clin Biochem 42: 1331-1346, 2009.

27. Liu F, Song YK and Liu D: Hydrodynamics-based transfection in animals by systemic administration of plasmid DNA. Gene Ther 6: 1258-1266, 1999.

28. McAnuff MA, Rettig GR and Rice KG: Potency of siRNA versus shRNA mediated knockdown in vivo. J Pharm Sci 96: 2922-2930, 2007.

29. Ventura-Sobrevilla J, Boone-Villa VD, Aguilar CN, et al: Effect of varying dose and administration of streptozotocin on blood sugar in male CD1 mice. Proc West Pharmacol Soc 54: 5-9, 2011.

30. Lewis DL and Wolff JA: Systemic siRNA delivery via hydrodynamic intravascular injection. Adv Drug Deliv Rev 59: 115-123, 2007.

31. Galic S, Hauser C, Kahn BB, et al: Coordinated regulation of insulin signaling by the protein tyrosine phosphatases PTP1B and TCPTP. Mol Cell Biol 25: 819-829, 2005. 